\title{
Gold Capsules for High Pressure Experiments
}

\author{
W. F. Wall \\ National Physical Laboratory, Teddington, England
}

The exceptional ductility and corrosion resistance of gold are utilised in the study of chemical reactions at very high pressures. To isolate the reactants from the pressure-transmitting fluid they are enclosed in sealed capsules which are constructed from thin-walled gold tubing.

At the National Physical Laboratory there is a facility for the generation of hydrostatic pressures up to $25 \mathrm{kbar}\left(25,000\right.$ atmospheres) and $250^{\circ} \mathrm{C}$ in piston-cylinder units, having a maximum compressed volume of $25 \mathrm{ml}$ (1). Fig. I shows a unit being lowered into its frame.

A single unit consists of a simple cylinder of high strength steel $\left(2000 \mathrm{MN} / \mathrm{m}^{2}\right)$, having an outside diameter to inside diameter ratio of approximately 7 . The cylinder is autofrettaged during its proving runs. This is effected by applying a pressure at the bore of the cylinder of such magnitude that the inner layers of steel in the cylinder are overstrained, passing from the elastic into the plastic range, and the plastic/elastic boundary is pushed some way into the cylinder wall. If the internal pressure within the cylinder is now released, the outer layers of the cylinder which were in elastic tension, now compress the inner layers which were plastic. The residual stress distribution in the cylinder wall is now very similar to that produced by a wirewound or a duplex "shrink-fit" vessel, with the advantage that external heating may be applied to the autofrettaged monobloc

\footnotetext{
Fig. 1 A piston-cylinder unit suitable for the generation of hydrostatic pressures up to 25 kbar $(25,000$ atmospheres) at up to $250^{\circ} \mathrm{C}$ being lowered into its frame at the National Physical Laboratory. The pressure is generated by forcing the piston into the cylinder. The reactant liquids are contained in gold capsules inside this cylinder
}

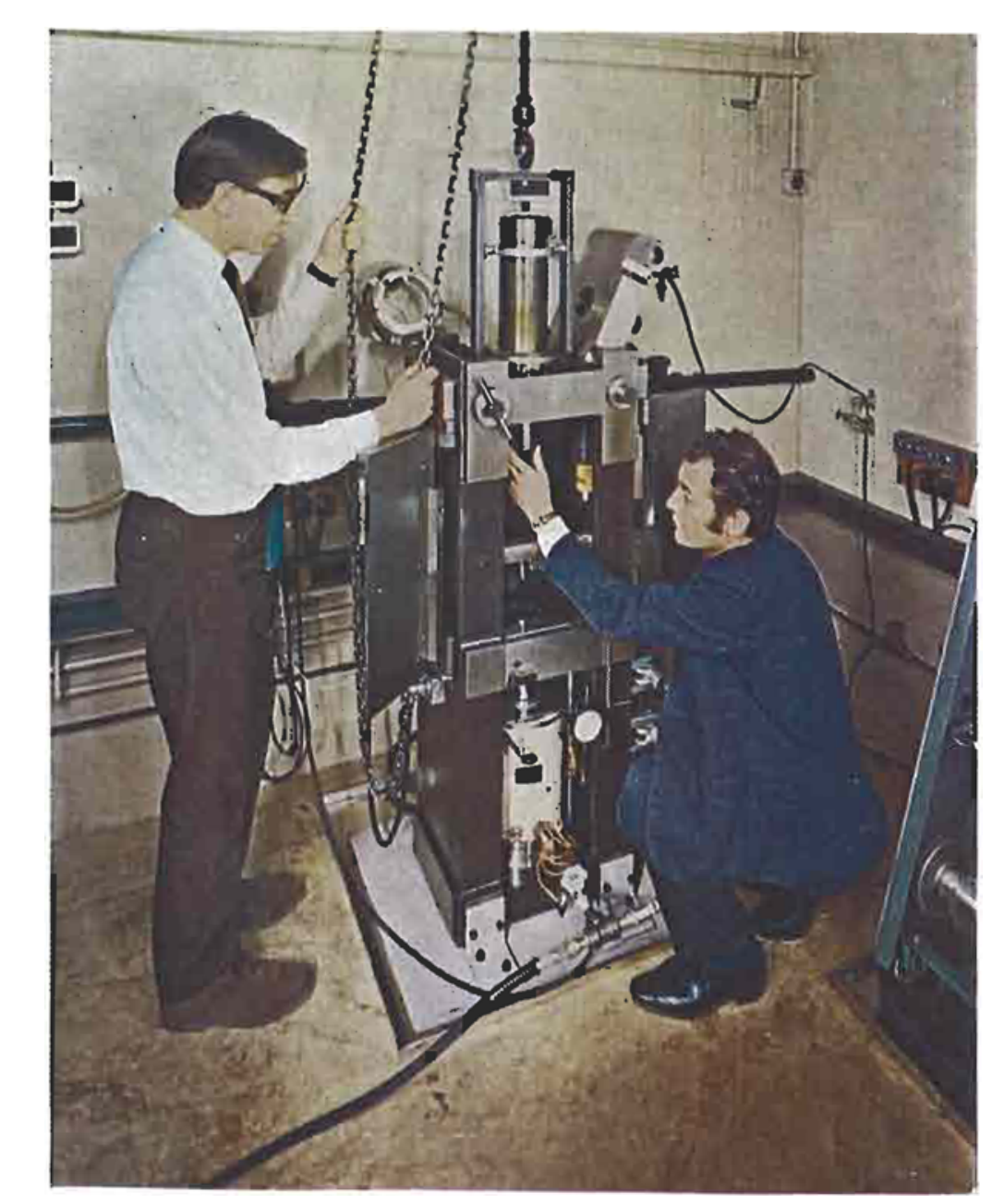

cylinder. External heating applied to a duplex vessel would markedly affect the interference and so the stress between the two concentric cylinders. With care, the application of autofrettage will double the pressure range for elastic behaviour of a thickwalled simple cylinder.

The cylinder is housed within a frame constructed from $300 \mathrm{~mm} \times 100 \mathrm{~mm}$ rolled steel channel, and pressure is generated within the cylinder by forcing a 
Fig. 2 Exploded view of a typical capsule assembly. Gold tubing has the ability to deform under higl pressure without rupturing. Gold is also fabricated easily into the capsule, it is inert to most reactants, and its higl melting point permits it to withstand exothermic reactions of the reactants

piston of super high speed steel into the bore of the cylinder, a $10 \mathrm{Mgf}$ ram providing the motive power. The cylinder bore is closed at the top by a high speed steel plug bear-

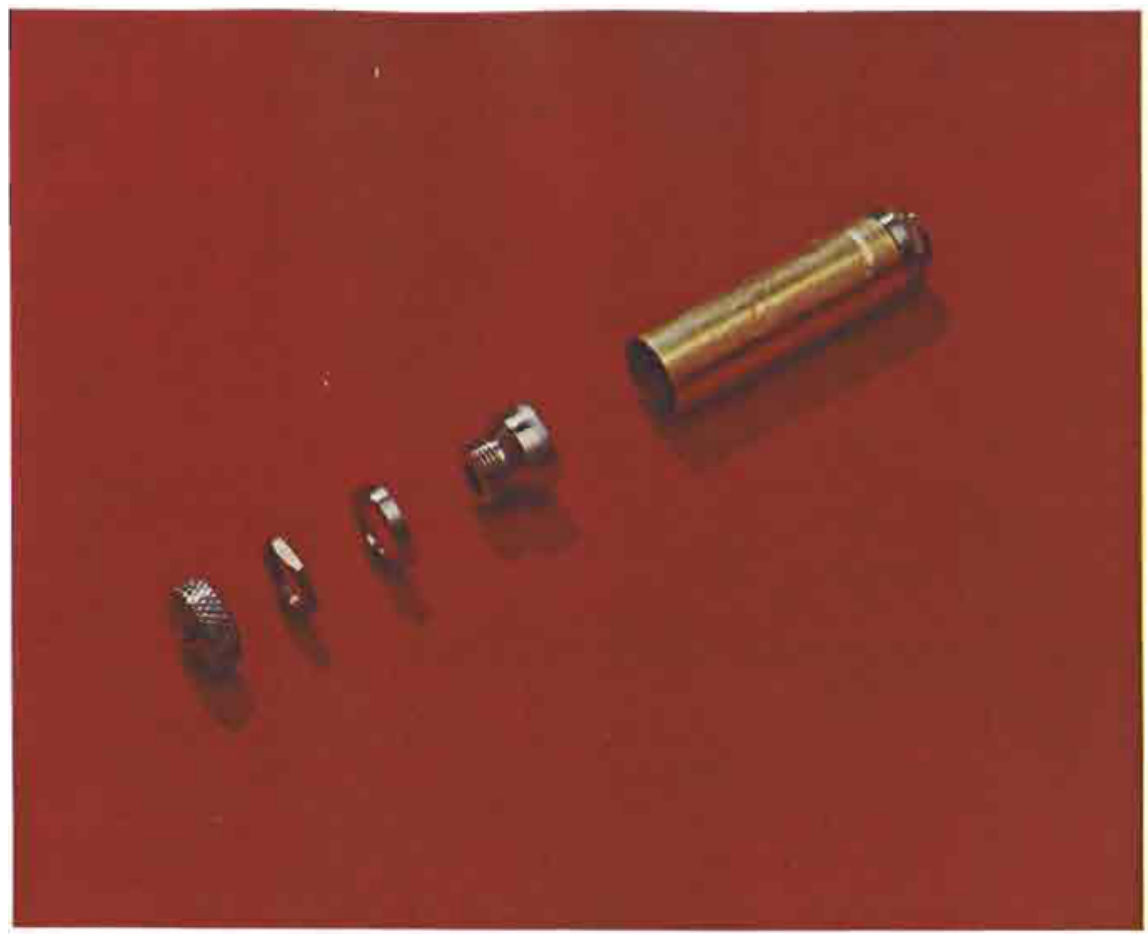
ing a polymeric O-ring seal backed by beryllium-copper anti-extrusion rings (2). A similar static seal is used at the bottom of the cylinder for the sliding piston.

A low-viscosity mineral oil is used as the pressuretransmitting medium within the high pressure environment. The system is instrumented such that pressure and temperature may be maintained at predetermined values for very long periods, and the parameters of pressure, temperature, and piston position are recorded continuously. The system is automatically shut down should any failure occur or should any parameter exceed a preset value.

The high pressure units are, and have been for some hundreds of runs, used for a wide variety of experiments, including pressure-soaking of metals, polymerisation studies (3) and chemical reactions under high pressure.

In most cases, and certainly in those involving chemical reactions, the reactants must be isolated

from the pressure-transmitting fluid. Thin-walled gold tubing has been used very successfully for this purpose, and a typical capsule assembly is shown in Figs. 2 and 3, and consists of a length of annealed gold tube 6 of wall thickness $0.15 \mathrm{~mm}$ which is spun on to stainless steel end closures 1 and 2, then locked on with a bevelled washer 3 and nut 4 . One end 2 is normally solid, while the other end is drilled so that the reactants may be introduced into the capsule from a hypodermic syringe. After filling, the filler hole is closed by a small gold ball 9 , held in place by a grub screw 8 via a steel ball bearing 10 . In practice it is prudent to rub three roughly equispaced flats on the circumference of the capsule to initiate where deformation of the gold tube will take place. Not only does the tube have to deform as the material within the capsule reduces in volume under pressure (this is up to 50 per cent of the original volume for organic liquids to $25 \mathrm{kbar}$ ), but the final

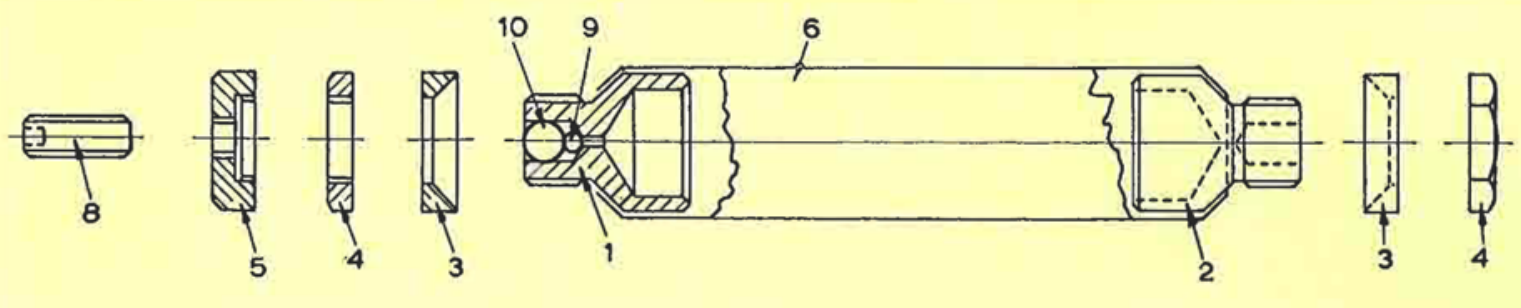

Fig. 3 The parts of a capsule assembly. The tube 6 and small ball closure 9 are made from gold. The end closures 1 and 2 , washers 3 , nuts 4 and 5 , grub serew 8 , and ball bearing 10 are all fabricated from stainless steel 
volume of the reacted material is normally less than the starting volume of the reactants. The overall strain accepted by the gold tube is therefore very considerable, and considerable deformation occurs to the capsule after a chemical reaction under high pressure.

These capsules have been tested to an internal pressure of 15 bar without rupture and may be used for some low boiling liquids, although for liquids having a vapour pressure of more than 2 bar at room temperature it is the practice to use $0.25 \mathrm{~mm}$ wall thickness gold tubing with 5 per cent nickel-gold solid ends brazed in as plugs, one end carrying a $1 \mathrm{~mm}$ bore $2 \mathrm{~mm}$ OD nickel filling tube. This nickel tube is attached to a vacuum line and the material to be pressed is vacuum-distilled into the capsule through the nickel tube. When the capsule is full, it is sealed by cold pinch-welding the nickel tube and finally "pinching-off" from the vacuum line.

The third type of capsule in use is much simpler but quite leak-tight and suitable for exploratory experiments requiring samples up to about $0.5 \mathrm{~g}$. For this, a 2 to $3 \mathrm{~cm}$ length of $5 \mathrm{~mm} \mathrm{OD} \times 0.1 \mathrm{~mm}$ wall thickness gold tubing is pinched flat at one end for about $5 \mathrm{~mm}$, folded over, and the fold covered by a small piece of $0.1 \mathrm{~mm}$ gold sheet. This is then placed in a slot in a short length of $3 \times 3 \mathrm{~mm}$ mild steel bar which is crushed in a vice on to the folded gold tube. After filling, the other end of the tube is treated in the same way.

The advantages of gold over other materials for the construction of these capsules have been (a) the workability, enabling spinning and clamping of the ends of the tube to be easily effected; $(b)$ the inertness of gold to most chemicals; $(c)$ the high melting-point of gold, enabling the product to be contained within the capsule should an exothermic reaction take place leading to an excessive temperature; $(d)$ the ductility and low shear yield stress allowing the large volume change of the capsule contents to be accommodated and giving a low pressure differential between the pressure transmitting fluid and the capsule contents; (e) the high reclaim value of the used gold tubing keeps the cost of each capsule to a reasonable figure.

The work described in this note forms part of the research programme of the High Pressure Section, Division of programme of the High Pressure Section, Division of
Inorganic and Metallic Structure, National Physical Laboratory.

\section{References}

1 H. S. Turner, W. F. Wall and S. L. S. Thomas, Nat. Phys. Lab. Report I.M.S. 16 (June 1972)

2 M. S. Paterson, f. Sci. Instrum., 1962, 39, 173

3 R. K. Bartlett, G. O'Neill, N. G. Saville, S. L. S. Thomas and W. F. Wall, Br, Polymer. F., 1970, 2, 225

\section{Diffusion Behaviour of Copper and Gold}

The sequential deposition of metallic thin films has become accepted as a perfectly routine procedure in the manufacture of microelectronic components. However, in terms of thermal stability, composite films of this nature must be considered as planar diffusion couples of exceptional thinness, with behaviour unfortunately differing considerably from that of bulk samples. As component reliability is of prime importance, it becomes necessary to learn as much as possible about the diffusion behaviour of thin films and, with this in mind, two investigators at I.B.M. undertook a quantitative study of interdiffusion between copper and gold (1).

Samples for investigation were prepared by the consecutive evaporation of each of these metals on to fused quartz substrates. They were subsequently examined by X-ray diffractometry both before and after annealing operations at various accurately controlled temperatures between 160 and $220^{\circ} \mathrm{C}$, for periods of up to 500 hours.

Previous work on bimetallic couples and, indeed, classical theory suggest that all phases in the diagram should appear in the annealed couples, though not necessarily all at the same time. Curiously enough only two new phases, $\mathrm{Cu}_{3} \mathrm{Au}$ and $\mathrm{CuAu}_{3}$, were found-the other possible ordered phase, $\mathrm{CuAu}$, being conspicuously absent. This perplexing observation was additionally confirmed by normal metallographic techniques which showed the annealed couple to have a layered structure of $\mathrm{Cu} / \mathrm{Cu}_{3} \mathrm{Au} / \mathrm{CuAu}_{3} / \mathrm{Au}$ with abrupt steps in composition between the different phases.

The absence of $\mathrm{CuAu}$ is something of an enigma, and the lack of any form of concentration gradient in the terminal metals is contrary to the established laws of intermetallic diffusion, and indeed has already evoked comment to this effect (2).

Furthermore, it is equally curious that $\mathrm{CuAu}_{3}$ appears as a separate layer, since its critical temperature for disordering is $200^{\circ} \mathrm{C}$, which lies well inside the upper temperature limit set for this experiment.

Nevertheless, although results show the growth of both $\mathrm{Cu}_{3} \mathrm{Au}$ and $\mathrm{CuAu}_{3}$ to be linear and their thickening rates identical, the rate is much too rapid to be due to volume diffusion and much too slow to be due to grain boundary diffusion. Logically, it may be concluded that if the growth rate is not governed by the rate of diffusion of copper and gold through the ordered layers, it must be some function of atomic movements at the interface, acting in such a way as to ensure both the correct order and stoichiometry. It thus begins to seem quite probable that formation of a $\mathrm{CuAu}$ layer was, in any case, impossible in the situation described.

A. D. M. K.

\section{References}

1 K. N. Tu and B. S. Berry, F. Appl. Phys, , 1972, 43, (8), 3283-3290

2 Nature Physical Science, 1972, 239, (91), 50 